\title{
Plasminogen promotes cholesterol efflux by the ABCA1 pathway
}

\author{
Nathalie Pamir, ${ }^{1}$ Patrick M. Hutchins, ${ }^{1}$ Graziella E. Ronsein, ${ }^{1}$ Hao Wei, ${ }^{1}$ Chongren Tang, ${ }^{1}$ Riku Das, ${ }^{2}$ \\ Tomas Vaisar, ${ }^{1}$ Edward Plow, ${ }^{2}$ Volker Schuster, ${ }^{3}$ Marlys L. Koschinsky, ${ }^{4}$ Catherine A. Reardon, ${ }^{5}$ \\ Richard Weinberg, ${ }^{6}$ David A. Dichek, ${ }^{1}$ Santica Marcovina, ${ }^{1}$ Godfrey S. Getz,' and Jay W. Heinecke' \\ 'Department of Medicine, University of Washington, Seattle, Washington, USA. ${ }^{2}$ Department of Molecular Cardiology, \\ Cleveland Clinic, Cleveland, Ohio, USA. ${ }^{3}$ Hospital for Children and Adolescents, Medical Faculty of Leipzig University, \\ Leipzig, Germany. ${ }^{4}$ Robarts Research Institute, Schulich School of Medicine and Dentistry, The University of Western \\ Ontario, London, Ontario, Canada. ${ }^{5}$ Ben May Research Institute, University of Chicago, Illinois, USA. ${ }^{6}$ Department of \\ Internal Medicine, Wake Forest School of Medicine, Winston-Salem, North Carolina, USA. 'Department of Pathology, \\ University of Chicago, Chicago, Illinois, USA.
}

Using genetic and biochemical approaches, we investigated proteins that regulate macrophage cholesterol efflux capacity (CEC) and ABCA1-specific CEC (ABCA1 CEC), 2 functional assays that predict cardiovascular disease (CVD). Macrophage CEC and the concentration of HDL particles were markedly reduced in mice deficient in apolipoprotein A-I (APOA1) or apolipoprotein $E$ (APOE) but not apolipoprotein A-IV (APOA4). ABCA1 CEC was markedly reduced in APOA1-deficient mice but was barely affected in mice deficient in APOE or APOA4. High-resolution size-exclusion chromatography of plasma produced 2 major peaks of ABCA1 CEC activity. The early-eluting peak, which coeluted with $\mathrm{HDL}$, was markedly reduced in APOA1- or APOE-deficient mice. The lateeluting peak was modestly reduced in APOA1-deficient mice but little affected in APOE- or APOA4deficient mice. Ion-exchange chromatography and shotgun proteomics suggested that plasminogen (PLC) accounted for a substantial fraction of the ABCA1 CEC activity in the peak not associated with HDL. Human PLG promoted cholesterol efflux by the ABCA1 pathway, and PLG-dependent efflux was inhibited by lipoprotein(a) [Lp(a)]. Our observations identify APOA1, APOE, and PLC as key determinants of CEC. Because PLG and Lp(a) associate with human CVD risk, interplay among the proteins might affect atherosclerosis by regulating cholesterol efflux from macrophages.

Conflict of interest: ). Heinecke is named as a coinventor on patents from the US Patent Office (patents are listed at the end of the supplemental material) on the use of HDL markers to predict the risk of cardiovascular disease and has served as a consultant for Kowa, Merck, Amgen, Bristol Meyer Squibb, CSK, and Pacific Biomarkers.

Submitted: December 9, 2016

Accepted: June 20, 2017

Published: August 3, 2017

\section{Reference information:}

JCI Insight. 2017;2(15):e92176. https://doi.org/10.1172/jci.

insight.92176.

\section{Introduction}

Clinical and epidemiological studies show a robust, inverse association of HDL cholesterol (HDL-C) levels with cardiovascular disease (CVD) risk $(1,2)$. Moreover, genetically engineered deficiencies in proteins implicated in HDL metabolism markedly affect atherosclerosis in hypercholesterolemic mice $(3,4)$, placing HDL in the causal pathway of accelerated atherogenesis in animal models. These observations have triggered intense interest in targeting HDL-C for therapeutic intervention.

Levels of HDL-C do not necessarily reflect the concentrations of cardioprotective HDL particles, however. $\mathrm{HDL}$ is a collection of macromolecules ranging in size from less than $7 \mathrm{~nm}$ to more than $14 \mathrm{~nm}(5)$, and the cholesterol content of the different species can vary 3-fold. Indeed, several lines of evidence suggest that the association between HDL-C levels and CVD status is indirect and that elevating HDL-C is not necessarily therapeutic (6-12). For example, genetic variations that associate with altered HDL-C do not strongly associate with altered CVD risk (9). Trials of drugs such as cholesteryl ester transfer protein inhibitors and niacin, which elevate HDL$\mathrm{C}$ by different mechanisms, have failed to reduce events in statin-treated subjects with established CVD (6-8, 10, 11). Moreover, a loss-of-function variant of scavenger receptor B1 (SCARB1) that raises HDL-C levels increases the risk of CVD in humans (13), and genetic deletion of SCARB1 in mice raises HDL-C levels and promotes atherosclerosis (14). These observations indicate that HDL-C does not necessarily reflect HDL's cardioprotective effects in either humans or mice and that it is critical to examine other functional properties of HDL.

HDL's cardioprotective effect is attributed in part to its ability to mobilize excess cholesterol from artery wall macrophages (15-18). The first key step involves ABCA1 - an ATP-binding cassette transporter found in the plasma membrane of macrophages - that exports phospholipids and cholesterol from 
cells (15-17). Lipid-poor apolipoprotein A-I (APOA1), HDL's major protein, is thought to be the dominant ligand for ABCA1 in vivo, but recent studies indicate that mature lipidated HDL particles also promote efflux by this pathway $(13-15,17,19,20)$.

Mouse and human studies have shown that defects in the APOA1/ABCA1 pathway are important determinants of CVD. For example, ablating the Abcal gene in myeloid cells increases atherosclerotic lesions in hypercholesterolemic mice (21), while overexpressing human ABCA1 in myeloid cells in hypercholesterolemic mice retards atherogenesis (22). Importantly, humans deficient in ABCA1 (Tangier's disease) accumulate cholesterol-laden macrophages in many different tissues despite having low levels of LDL-cholesterol, providing strong evidence that ABCA1 conducts a key step in sterol excretion from these cells in humans (23).

Cholesterol efflux capacity (CEC) - the ability of human serum depleted of lipoproteins containing apolipoprotein B (APOB) to promote cholesterol efflux from macrophages - can vary markedly between different subjects despite similar levels of HDL-C and APOA1 (24). Macrophage CEC of serum HDL associated strongly and negatively with prevalent CVD status in 2 large, independent human populations (25). That association was independent of HDL-C and APOA1 levels. CEC inversely associated with future cardiac events in the Dallas Heart and the EPIC-Norfolk studies $(26,27)$. These relationships remained highly significant after correction for other classic lipid risk factors for CVD. The assay in the Dallas Heart Study monitored cholesterol efflux capacity specifically by the ABCA1 pathway (28), while the EPIC-Norfolk Study quantified macrophage CEC (24), which involves ABCA1 and multiple other lipid transporters. Taken together, these observations provide strong evidence that macrophage $\mathrm{CEC}$ and ABCA1 CEC are clinically relevant metrics of HDL function that are independent of HDL-C.

The mechanisms that mediate CEC in APOB-depleted serum are poorly understood. In the current study, we used mice deficient in HDL apolipoproteins implicated in cholesterol efflux from macrophages $(15,16,29)$ and analyzed plasma samples biochemically to investigate those proteins' roles in macrophage CEC and ABCA1 CEC. Our observations indicate that APOA1, apolipoprotein E (APOE), and plasminogen (PLG, which is not an apolipoprotein) are key modulators of CEC, and that PLG-dependent ABCA1 CEC is inhibited by lipoprotein(a) [Lp(a)]. These findings could provide the basis for studies of factors that influence CEC — and therefore cardioprotection — in vivo.

\section{Results}

We quantified CEC with both serum HDL (serum depleted of lipoproteins containing APOB) and plasma fractionated by high-resolution size-exclusion chromatography (SEC). Because serum HDL contains mature HDL as well as partially lipidated and lipid-free forms of APOA1 and apolipoprotein A-IV (APOA4), fractionating the plasma by SEC allowed us to distinguish between HDL-associated and nonHDL-associated proteins that promote CEC. We complemented these studies by quantifying HDL particle concentration (defined as lipoproteins isolated by ultracentrifugation at $d=1.063-1.21 \mathrm{~g} / \mathrm{ml}$ ) in the different strains of mice.

Both $A P O A 1$ and $A P O E$ are important regulators of macrophage $C E C$, but only APOA1 promotes ABCA1 CEC. Using serum HDL from wild-type mice or mice deficient in APOA1, APOE, APOA1/APOE, or APOA4 (all in the C57BL/6 genetic background), we determined whether these proteins modulate CEC and/or HDL particle concentration. Macrophage CEC, which involves multiple lipid transporters $(30,31)$, was monitored with cAMP-stimulated J744 macrophages (24, 25); ABCA1 CEC, which involves only ABCA1, was quantified with BHK cells with inducible expression of human ABCA1 (32).

Macrophage CEC was markedly lower in mice deficient in APOA1 or APOE than in wild-type mice (Figure $1 \mathrm{~A} ; P<0.0001)$. It was even lower $(\sim 80 \% ; P<0.0001)$ in mice deficient in both proteins. In contrast, APOA4 deficiency had little effect on macrophage CEC $(33,34)$. ABCA1 CEC was likewise reduced in APOA1- and APOA1/APOE-deficient mice (Figure 1B; $P=0.003$ and $P=0.002$, respectively), but the effect was smaller ( $\sim 50 \%)$ than that observed for macrophage CEC $(\sim 70 \%)$. However, ABCA1 CEC was barely affected in mice deficient in only APOE or only APOA4.

Phospholipid and cholesterol levels were markedly reduced in serum HDL of mice deficient in APOA1, APOE, or APOA1/APOE (Figure 2, A and B), as reported for HDL-C levels in these animals (35-37). There were no significant changes in phospholipids or cholesterol in serum HDL of mice deficient in APOA4 (Figure 2, A and B), suggesting that HDL-C levels were not significantly lower than in wild-type mice. 

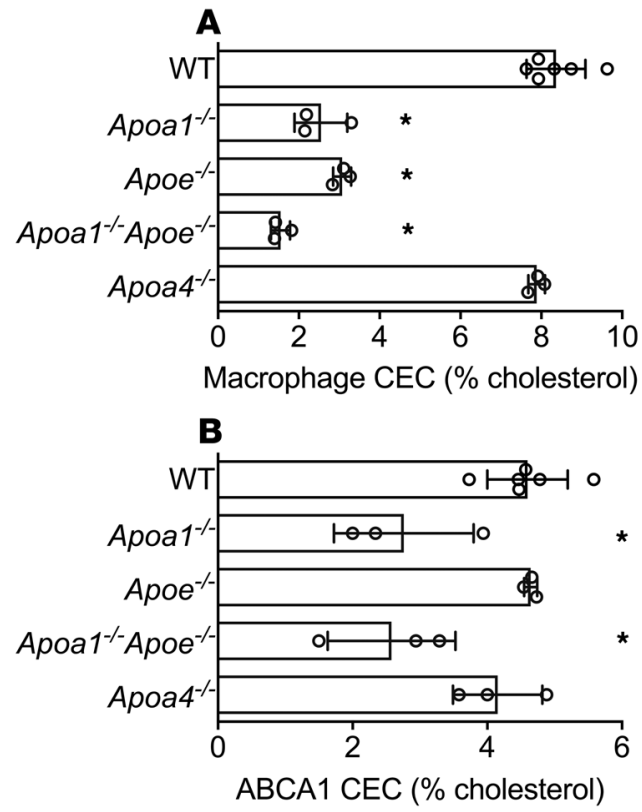

Figure 1. Macrophage cholesterol efflux capacity (CEC) and ABCA1-specific CEC (ABCA1 CEC) for wild-type mice and mice deficient in APOA1, APOE, APOA1/APOE, or APOA4. Serum HDL (APOB-depleted serum) was obtained by polyethylene glycol precipitation of plasmaderived serum of wild-type mice (WT, $n=6$ ), Apoa1-/mice $(n=6)$, Apoe $e^{-/-}$mice $(n=6)$, Apoa1 $^{-1-} ;$ Apoe $^{-/-}$mice $(n=3)$, or Apoa $^{-/-}$mice $(n=3)$. (A) Macrophage CEC and (B) ABCA1 CEC of serum HDL were monitored using $\left[{ }^{3} \mathrm{H}\right]$ cholesterol-labeled $\mathbf{7 7 4}$ macrophages and baby hamster kidney (BHK) cells, respectively. Cholesterol efflux was calculated as the percentage of radiolabel in the medium of the cells at the end of a 4-hour incubation divided by the total radioactivity of the medium and cells. Macrophage CEC and ABCA1 CEC were quantified as the difference in cholesterol efflux of cells with and without exposure to CAMP and with or without induction of ABCA1 expression, respectively. Significance was determined by ANOVA followed by a Fisher's post-hoc analysis for multiple comparisons. ${ }^{*} P<0.05$ versus wild-type mice.

Because the concentration of HDL particles might have important effects on CEC, we quantified HDL particle number by calibrated ion mobility analysis (HDL- $\mathrm{P}_{\text {IMA }}$; see ref. 38), using HDL isolated by ultracentrifugation from plasma of the different mouse strains (Figure 2C). HDL- $\mathrm{P}_{\text {IMA }}$ was lower in mice deficient in APOA1 (by 70\%) or APOE (by 50\%) than in wild-type mice. Moreover, mice deficient in both APOA1 and APOE almost completely lacked HDL particles (98\% decrease). In contrast, there was no significant change in HDL- $\mathrm{P}_{\mathrm{IMA}}$ in mice deficient in APOA4. Serum HDL cholesterol and phospholipid levels were similar in mice deficient in APOA1 or APOE, but HDL particle concentration was lower in the APOA1-deficient mice, suggesting that those mice have fewer larger particles.

We next quantified the relationship between HDL-P ${ }_{\text {IMA }}$ and macrophage CEC or ABCA1 CEC in all the mouse strains. While HDL- $\mathrm{P}_{\text {IMA }}$ and macrophage CEC correlated strongly $(r=0.72, P=0.0007)$, HDL- $\mathrm{P}_{\text {IMA }}$ did not correlate with ABCA1 CEC $(r=0.18, P=0.47)$, suggesting that factors independent of HDL particle concentration (lipoproteins of $d=1.063-1.21 \mathrm{~g} / \mathrm{ml}$ ) can mediate cholesterol efflux by the ABCA1 pathway.

Collectively, these observations indicate that both APOA1 and APOE are important mediators of macrophage CEC but that only APOA1, likely as a lipid-poor apolipoprotein (17), promotes ABCA1 CEC. In contrast, ABCA1 CEC was much less affected in mice with APOA1 and/or APOE deficiency. Moreover, APOA4 was not a major contributor to macrophage CEC or ABCA1 CEC. We previously showed that ABCA1 CEC is not reduced in APOE-deficient mice despite the marked reductions in HDL- $\mathrm{P}_{\text {IMA }}$, HDL-C, and cholesterol and phospholipid levels in serum HDL (39), suggesting that proteins or factors other than HDL's major proteins and HDL particle concentration are important determinants of ABCA1 CEC.

A peak of material not associated with APOA1 promotes ABCA1 CEC. To investigate the molecular basis for the differences between macrophage CEC and ABCA1 CEC in the different strains of mice, we quantified CEC in fractions isolated from EDTA plasma by high-resolution SEC (Figure 2 and Supplemental Figure 2; supplemental material available online with this article; https://doi.org/10.1172/jci.insight.92176DS1). Under these conditions, the large APOB-containing lipoproteins (VLDL and LDL) elute with a different retention time from the smaller lipidated HDL particles. We used plasma rather than serum HDL because preliminary studies demonstrated that polyethylene glycol, the material used to precipitate APOB-containing lipoproteins from serum, affects HDL's migration during SEC.

APOA1 eluted as a single major peak in fractions 8-16 of plasma (the apparent size of mature, lipidated HDL) when it was isolated from wild-type mice (Figure 2A and Supplemental Figure 1). A small shoulder of APOA1, likely lipid-free or poorly lipidated APOA1, eluted later (fractions 17-23). Both cholesterol and phospholipids also eluted as a major peak with a small shoulder (which contained phospholipid but very little cholesterol) that coeluted with APOA1 (Figure 2, B and C, and Supplemental Figure 1C). 

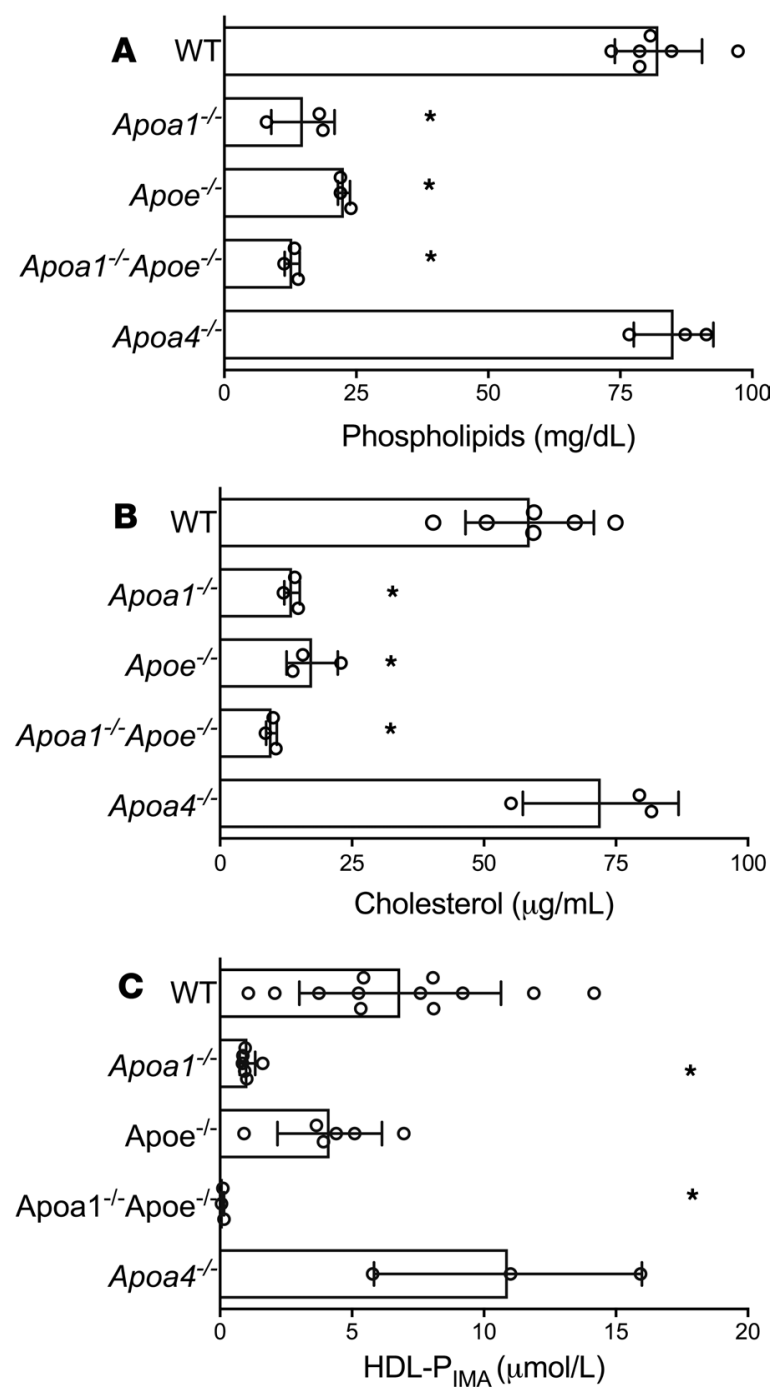

Figure 2. Plasma phospholipid level, plasma cholesterol level, and HDL particle concentration (HDL- ${ }_{\text {IMA }}$ ) of wild-type mice and mice deficient in APOA1, APOE, APOA1/APOE, or APOA4. Phospholipid (A) and total cholesterol (B) levels were quantified biochemically. (C) HDL- $P_{\text {IMA }}$ was determined by calibrated ion mobility analysis using glucose oxidase as the standard. Significance was determined by ANOVA followed by Fisher's posthoc analysis for multiple comparisons. ${ }^{*} P<0.05$ versus wild-type mice (WT).

We next quantified the CEC of fractions isolated by SEC from the 5 strains of mice (Figure 2 and Supplemental Figure 2). The fractions of wild-type mouse plasma that promoted macrophage CEC eluted as a single major peak of material that coeluted with the apparent size of HDL (Figure 2A and Supplemental Figure 2A). In striking contrast, plasma exhibited 2 major peaks of ABCA1 CEC activity. One coeluted with the apparent size of HDL, while the other eluted later and had an apparent size smaller than that of HDL (Figure 2B and Supplemental Figure 2B).

To determine the role of APOA1 in CEC, we fractionated plasma from mice deficient in the protein (Figure 2, C and D, and Supplemental Material). Macrophage CEC was reduced by $70 \%$ in the peak of material that coeluted with the size of HDL. Macrophage CEC was also smaller in the small shoulder of the early-eluting peak of activity, suggesting that lipidfree APOA1 promotes cholesterol efflux.

ABCA1 CEC was also reduced ( $50 \%)$ in the major peak of material that coeluted with the size of HDL. However, there was only a modest reduction of ABCA1 CEC in the late-eluting peak of material (Figure 2D). Taken together, these observations indicate that HDL particles containing APOA1 are major mediators of macrophage CEC but that proteins or other factors not associated with APOA1 are also important contributors to ABCA1 CEC.

We next investigated the effects of APOE deficiency and APOA1/APOE deficiency on macrophage CEC and ABCA1 CEC (Figure 2, E-H, and Supplemental Figure 2). The peak with macrophage CEC activity that coeluted with the apparent size of HDL was approximately $30 \%$ smaller in the APOE-deficient mice than in the wild-type mice. ABCA1 CEC activity was lower in the peak of material with the apparent size of HDL but was not decreased in the late-eluting material (Figure 2, E and F, and Supplemental Figure 2, $\mathrm{E}$ and $\mathrm{F}$ ). These observations show that lipoproteins with the apparent size of HDL that contain APOE can also promote ABCA1 CEC. In contrast, APOE deficiency had no effect on the ABCA1 CEC activity of the material whose apparent size was smaller than that of mature HDL.

When we fractionated plasma from the APOA1/APOE-deficient mice by SEC (Figure 2G and Supplemental Figure 2G), the activity in the peak with the apparent size of HDL was markedly lower (90\%) than in the wild-type mice. The peak also had little ABCA1 CEC activity (Figure $2 \mathrm{H}$ and Supplemental Figure 2H), suggesting that HDL containing APOA1 and/or APOE promotes efflux mainly by the ABCA1 pathway. However, the ABCA1 CEC activity of the late-eluting peak was only modestly lower than the activity in the late-eluting peak of the wild-type mice (Figure $2 \mathrm{H}$ and Supplemental Figure $2 \mathrm{H}$ ). Collectively, these observations indicate that HDL particles containing APOA1 and/or APOE account 
almost quantitatively for macrophage CEC, but that other proteins or factors with an apparent molecular size smaller than HDL are major contributors to the ABCA1 CEC activity of plasma.

Albumin does not account for the late-eluting peak of ABCA1 CEC activity. Albumin and the late-eluting peak of material with ABCA1 CEC activity exhibited similar apparent sizes on SEC. However, albumin promotes shuttling of cholesterol from cells to medium when the medium is supplemented with high concentrations of exogenous cholesterol acceptor such as LDL, phospholipid vesicles, or red blood cells (40). Two lines of evidence suggest that albumin was not responsible for the late-eluting peak of ABCA1 CEC activity we observed in plasma fractionated by SEC. First, we do not include exogenous cholesterol acceptors in our CEC assays, and in our hands albumin does not promote cholesterol efflux by BHK cells expressing ABCA1.

Second, when we subjected mouse plasma of wild-type mice or APOA1-deficient mice to antibodybased affinity chromatography, albumin was quantitatively removed from the fractions containing the late-eluting ABCA1 CEC activity (Figure 3 and Supplemental Figure 3). However, HDL particles were also almost quantitatively lost from the albumin-depleted material, likely because of removal of APOA1. Previous work has shown that APOA1 coisolates with albumin during 4 independent methods of biochemical fractionation, including affinity isolation with antibodies (41). Consistent with these data, the peak of HDL-associated macrophage CEC was also markedly reduced. In striking contrast, the ABCA1 CEC activity of the late-eluting peak was reduced by only approximately $50 \%$, likely because of the loss of poorly lipidated APOA1.

Together, these observations strongly suggest that albumin is not a major contributor to the ABCA1 CEC activity we observe in the late-eluting peak of material.

PLG correlates with ABCA1 CEC in plasma subjected to orthogonal chromatography-mass spectrometry. We used orthogonal chromatography-mass spectrometry to identify non-HDL-associated proteins whose elution patterns correlated with those of ABCA1 CEC. Plasma from wild-type mice or APOA1-deficient mice were subjected to high-resolution SEC, and each fraction was monitored for ABCA1 CEC. Fractions from the late-eluting peak of material with ABCA1 CEC activity were combined and then separated by cationexchange (Figure 4, A and E) or anion-exchange (Figure 4, C and G) chromatography; each fraction was again assessed for ABCA1 CEC activity. The active fractions were analyzed with shotgun proteomics (42), and the relative concentrations of proteins (quantified as spectral counts, the number of unique peptides detected by MS/MS (43) in each fraction were correlated with ABCA1 CEC activity.

Hierarchical clustering assessed by Euclidean distance analysis revealed an array of proteins linked to ABCA1 CEC in the fractions obtained by each of the 2 methods. However, the proteins in the 2 clusters were distinct, and albumin was not in either cluster. Only 1 protein — plasminogen (PLG) - correlated strongly with ABCA1 CEC in both clusters. Moreover, only PLG correlated with ABCA1 CEC activity when plasma was first fractionated by SEC and then by cation-exchange (Figure 4, B and F) or anion-exchange (Figure 4, D and $\mathrm{H}$ ) chromatography (cation exchange, $r=0.81$ and $r=0.84$ for wild-type and Apoa ${ }^{-/-}$mice, respectively; anion exchange, $r=0.98$ and $r=0.95$ for wild-type and Apoa $1^{-1-}$ mice, respectively).

PLG stimulates cholesterol efflux from macrophages by the ABCA1 pathway. We exposed BHK cells (with and without inducible expression of human ABCA1), macrophages (with and without cholesterol loading), and bone marrow-derived macrophages (with and without expression of ABCA1) to human PLG (Figure 5 ) to determine whether the protein would promote cholesterol efflux from cells. SDS-PAGE analysis with Coomassie blue staining indicated that material with the apparent MW of PLG accounted for greater than $95 \%$ of the protein in the PLG preparation.

Stimulation by PLG of ABCA1-specific cholesterol efflux from BHK cells was half-maximal at approximately $0.7 \mu \mathrm{g} / \mathrm{ml}$ and plateaued at $3 \mu \mathrm{g} / \mathrm{ml}$ (Figure 4A). In contrast, PLG failed to promote cholesterol efflux from BHK cells expressing ABCG1 (Supplemental Figure 6) (44). Although PLG is a serine protease, its ABCA1 activity was not affected by phenylmethylsulfonyl fluoride (PMSF), which covalently modifies reactive serine residues (45). Cholesterol accumulation in the medium of cells exposed to $1 \mu \mathrm{g} / \mathrm{ml}$ PLG was linear for 2 hours but then gradually decreased (Figure 4B). In contrast, higher concentrations of APOA1 were required for half-maximal and maximal ABCA1-specific cholesterol efflux from BHK cells (Figure 4C): at $5 \mu \mathrm{g} / \mathrm{ml}$ APOA1, the amount of cholesterol in the medium of cells was approximately $30 \%$ higher than when cells were exposed to PLG. These results indicate that PLG stimulates cholesterol efflux from BHK cells that express lower concentrations of human ABCA1 than APOA1 but that APOA1 can promote greater total efflux than PLG in this model system. 


\section{WT Mice}
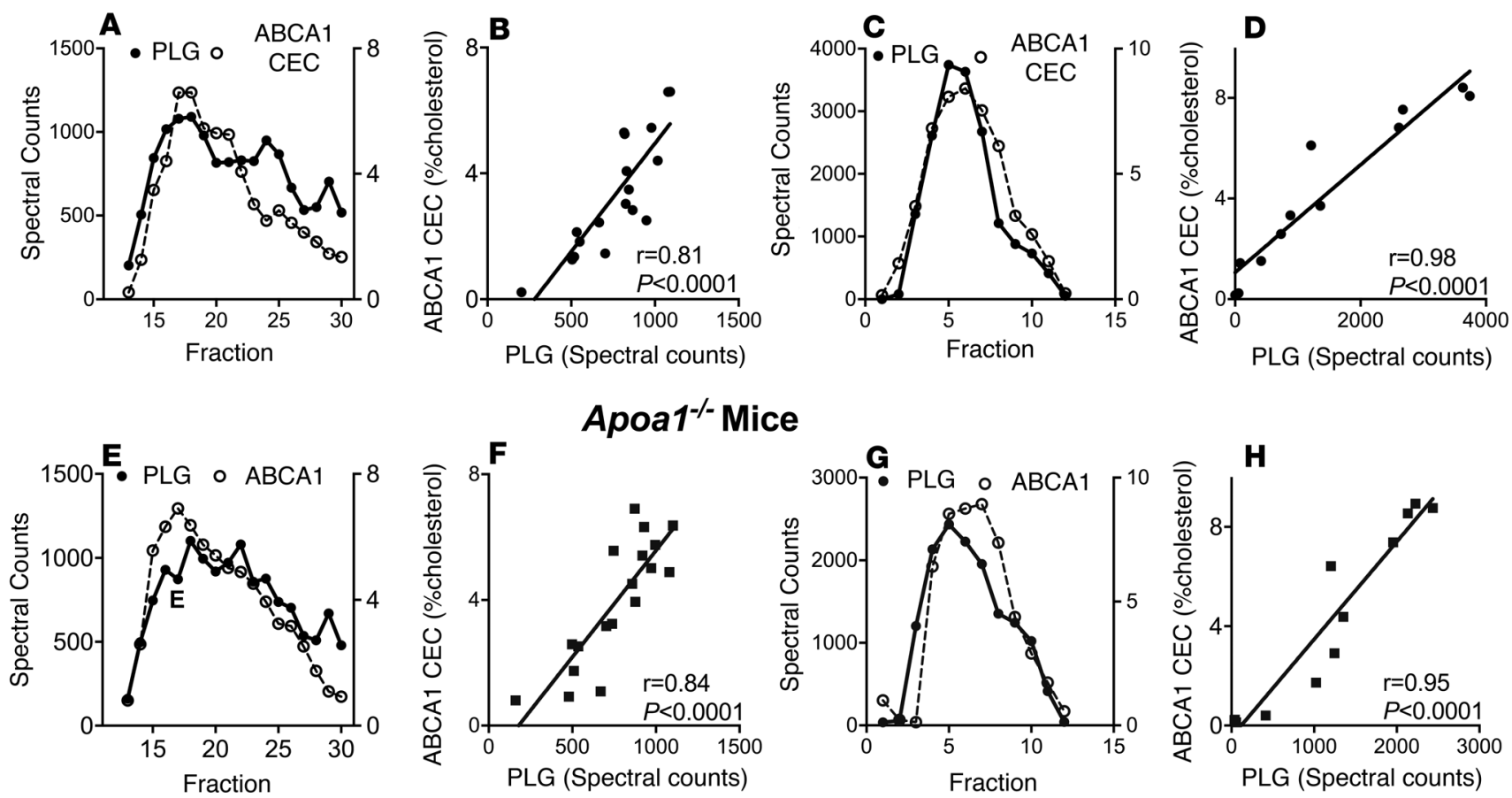

Figure 3. Tandem mass spectrometric analysis of proteins and quantification of ABCA1 CEC activity in mouse plasma fractionated by sequential high resolution SEC and ion-exchange chromatography. Plasma $(300 \mu \mathrm{l})$ of wild-type mice or Apoa $1^{-1-}$ mice was fractionated by size-exclusion chromatography (SEC) on a Superdex 200 column at a flow rate of $0.35 \mathrm{ml} / \mathrm{min}$. The late-eluting peak of material with ABCA1 cholesterol efflux capacity (CEC) activity (fractions 15-23) from 3 different animals was pooled, concentrated, and then subjected to cation-exchange (A and $\mathbf{E}$ ) or anion-exchange (C and $\mathbf{G}$ ) chromatography. ABCA1 CEC was measured for each fraction. Proteins in each fraction were quantified by tandem MS analysis with spectral counting (the number of unique peptides detected for each protein). Lines represent Pearson's correlation between ABCA1 CEC, and spectral counts in each fraction (B, D, F, and $\mathbf{G}$ ).

To determine whether PLG would also promote cholesterol efflux from macrophages, we used mouse thioglycolate-elicited peritoneal macrophages. PLG $(8 \mu \mathrm{g} / \mathrm{ml})$ increased the mass of cholesterol in medium of cholesterol-loaded peritoneal macrophages but not control peritoneal macrophages (Figure 4D). We observed similar results with APOA1 $(20 \mu \mathrm{g} / \mathrm{ml})$.

To explore the role of ABCA1 in PLG-mediated cholesterol efflux from macrophages, we isolated bone marrow-derived macrophages from wild-type $\left(\mathrm{Abcal}^{+/+}\right)$mice and mice partially $\left(\mathrm{Abca1}^{+/-}\right)$or completely $\left(\mathrm{Abca1}^{1^{-}-}\right)$deficient in ABCA1. Both PLG and APOA1 promoted cholesterol efflux into the medium of macrophages isolated from the wild-type mice (Figure 4E). However, cholesterol efflux was almost completely abrogated when the macrophages were deficient in $\mathrm{ABCA1}$.

Mouse and human plasma deficient in PLG have diminished ABCA1 CEC. To determine whether PLG might contribute to $\mathrm{ABCA} 1 \mathrm{CEC}$ in mice, we subjected pooled plasma from wild-type mice and PLG-deficient mice to high-resolution SEC and quantified ABCA1 CEC activity in fractions eluted from the column. The amount of ABCA1 CEC activity in the early-eluting peak (with the apparent size of HDL) was almost identical in the wild-type and PLG-deficient mice. In contrast, the amount of ABCA1 CEC activity in the late-eluting peak was approximately 50\% lower in PLG-deficient mouse plasma relative to that of wild-type mice.

We next determined whether plasma from PLG-deficient mice in the Apoe ${ }^{-/}$genetic background also was deficient in ABCA1 CEC activity. ABCA1 CEC was significantly lower in serum HDL of PLG-deficient $A_{p o e^{-/-}}$mice than in Apoe $e^{-/-}$mice $(10.5 \% \pm 0.5 \%$ and $12.3 \% \pm 0.4 \%$ cholesterol, respectively; $P=$ $0.025, n=5$ ). When the plasma of the 2 different strains of mice was pooled and subjected to SEC, the amount of ABCA1 CEC activity in the early-eluting peak (with the apparent size of HDL) was almost identical in the Apoe ${ }^{-/-}$and PLG-deficient Apoe $e^{-/}$mice. In contrast, the amount of ABCA1 CEC activity in the late-eluting peak was approximately $30 \%$ lower in PLG-deficient $A p o e^{--}$mouse plasma than in plasma of $A p o e^{-/-}$mice (Figure 4 and Supplemental Figure 4). Taken together, these observations suggest that PLG accounts for $30 \%-50 \%$ of the ABCA1 CEC activity in the late-eluting peak of activity in mouse plasma. 

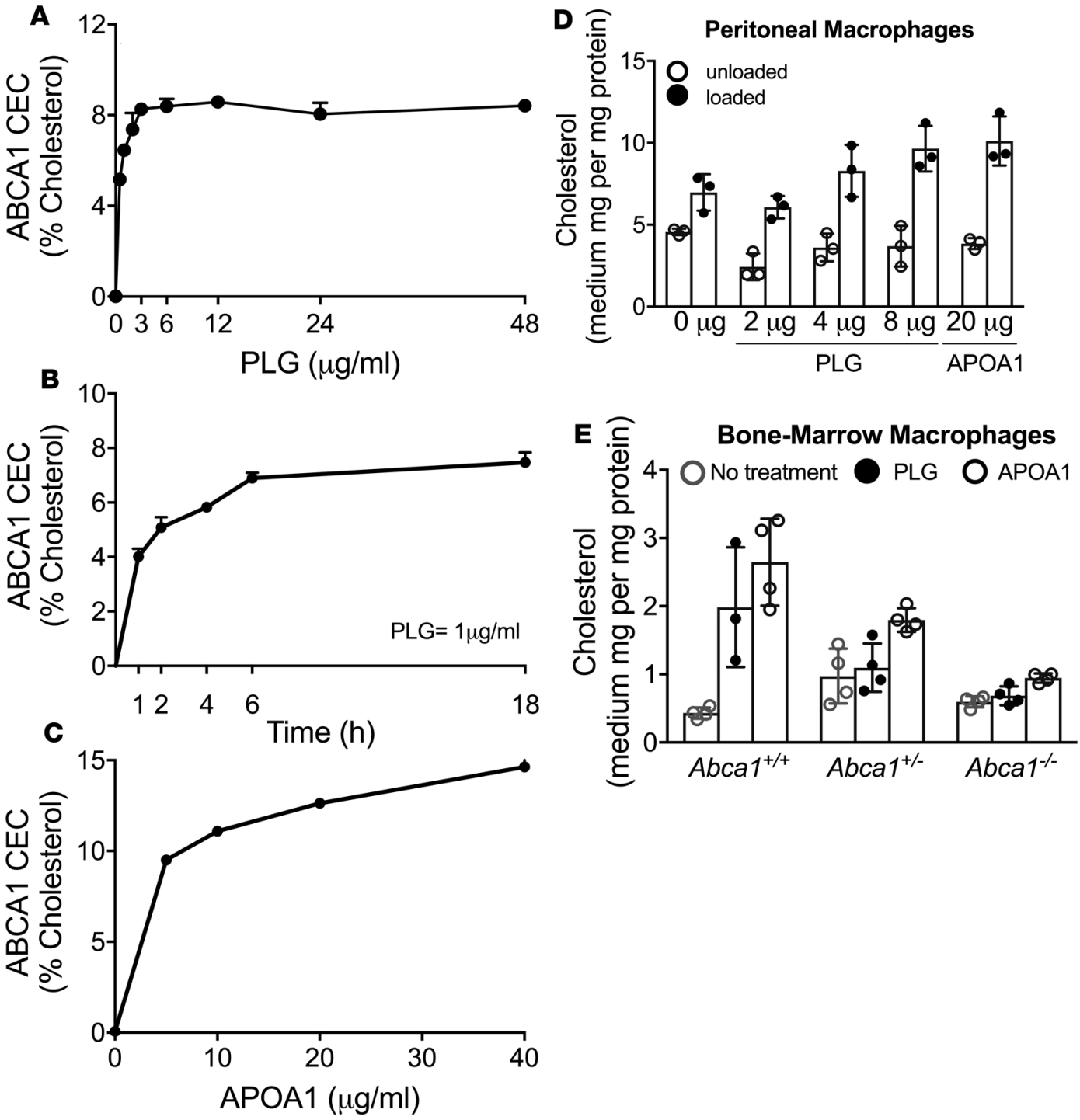

Figure 4. Quantification of plasminogen's (PLG's) ability to promote radiolabeled cholesterol efflux from ABCA1-expressing BHK cells and cholesterol accumulation in medium of macrophages. (A-C) Cholesterol efflux capacity was quantified using [ $\left.{ }^{3} \mathrm{H}\right]$ cholesterol-labeled BHK cells with or without induced expression of human ABCA1. Cells were incubated with the indicated concentrations of PLG or APOA1 in serum-free medium for 4 hours. Cholesterol efflux was calculated as the percentage of radiolabel in the medium of the cells at the end of the incubation divided by the total radioactivity of the medium and cells. ABCA1-specific cholesterol efflux was monitored as the difference in cholesterol efflux of cells with and without induction of ABCA1. (D) Macrophages were harvested from peritonea of wild-type mice 4 days after thioglycolate injection. (E) Macrophages were derived using M-CSF from bone marrow cells of wild-type $\left(\mathrm{AbCa}^{+/+}\right)$mice, mice heterozygous for ABCA1 expression $\left(\mathrm{Abca}^{+/-}\right)$, or mice deficient in ABCA1 (Abca1-/-). Macrophages were loaded with cholesterol by incubating them with $2 \%$ serum from LDL receptor-deficient mice that were fed a high-fat high-cholesterol diet for 16 weeks. ( $\mathbf{D}$ and $\mathbf{E}$ ) Cholesterol accumulation in the medium of macrophages was quantified by mass spectrometric analysis after a 4-hour incubation. CEC, cholesterol efflux capacity.

To determine whether PLG might contribute to ABCA1 CEC in humans, we chromatographed human plasma on L-lysine Sepharose (to which PLG binds with high specificity and affinity; see ref. 46). Immunoblot analysis with a monoclonal antibody confirmed that PLG was undetectable in the chromatographed material. When we measured macrophage CEC and ABCA1 CEC on the material that flowed through the column (Figure 5C), we found that PLG depletion significantly reduced $(P<0.05)$ both macrophage CEC and ABCA1 CEC. The magnitude of the reduction in ABCA1 CEC suggested that PLG was responsible for approximately $30 \%$ of total ABCA1 CEC in human plasma. Supplementing PLG-depleted serum with purified PLG $(4 \mu \mathrm{g} / \mathrm{ml})$ partially restored macrophage CEC but almost completely restored ABCA1 CEC (Figure 5C), suggesting that PLG contributes to cholesterol efflux by ABCA1 in this model system. 
Apolipoprotein A and Lp(a) inhibit PLG-dependent cholesterol efflux by the ABCA1 pathway. Apolipoprotein A (LPA) covalently binds to APOB on LDL particles, forming a unique atherogenic lipoprotein termed $\mathrm{Lp}$ (a) (47). Because LPA has high structural homology with PLG, we investigated its interactions with the ABCA1 pathway. Over a wide range of concentrations $(0.1-6 \mu \mathrm{g} / \mathrm{ml})$, recombinant human LPA (containing 17 kringle domains) failed to stimulate ABCA1-specific cholesterol efflux from BHK cells (Figure 6A). However, it markedly inhibited the ABCA1-specific cholesterol efflux (Figure 6A) induced by PLG (1 $\mu \mathrm{g} /$ $\mathrm{ml}$ ). Under those conditions, ABCA1-specific cholesterol efflux was inhibited half-maximally by approximately $0.6 \mu \mathrm{g} / \mathrm{ml} \mathrm{LPA}$.

Because all LPA in human plasma is present in Lp(a) particles, we next tested the effect of isolated Lp(a) on ABCA1-specific cholesterol efflux from BHK cells (Figure 6B). Lp(a) potently inhibited the ability of PLG $(1 \mu \mathrm{g} / \mathrm{ml})$ - but not APOA1 $(10 \mu \mathrm{g} / \mathrm{ml})$ - to promote ABCA1-specific cholesterol efflux from BHK cells. Inhibition was concentration-dependent, and was half-maximal at approximately $0.8 \mu \mathrm{g} / \mathrm{ml}$ of $\mathrm{Lp}(\mathrm{a})$.

\section{Discussion}

Using a genetic approach, we showed that HDL-associated APOA1 and APOE, but not APOA4, are important determinants of macrophage CEC. In contrast, APOA1, but not APOE or APOA4, was a key mediator of ABCA1 CEC. Biochemical fractionation revealed 1 major peak of macrophage CEC activity but 2 major peaks of ABCA1 CEC activity in plasma: an early-eluting peak with the apparent size of HDL and a late-eluting peak of material with an apparent size similar to albumin. HDL-associated APOA1 and/ or APOE accounted quantitatively for the early-eluting peak of macrophage CEC activity, but the second peak of late activity was distinct from HDL, APOA1, and APOE. A search for proteins in the late-eluting peak demonstrated that PLG also promotes cholesterol efflux from BHK cells that express ABCA1.

To determine whether PLG can regulate macrophage cholesterol homeostasis, we quantified cholesterol mass in medium of mouse macrophages. PLG promoted the accumulation of cholesterol mass in medium of cholesterol-loaded macrophages. With bone marrow-derived macrophages, cholesterol accumulation in the medium was dependent on both ABCA1 and PLG.

Collectively, these observations suggest that HDL-associated APOA1 and APOE are the major mediators of macrophage CEC but that other factors promote cholesterol efflux by the ABCA1 pathway. Our biochemical, cell culture, and genetic studies identified one such factor as PLG, a protein linked with atherosclerotic disease risk in multiple genetic studies (48-51). Significantly, Lp(a) and LPA blocked the ABCA1 CEC activity of PLG, but not that of APOA1. These observations suggest that the interplay between PLG and Lp(a) could be a key regulator of macrophage cholesterol efflux and foam cell formation in the artery wall. In future studies, it will be important to determine PLG's impact on Lp(a)-mediated delivery of cholesterol to macrophages and to ascertain whether PLG mediates HDL biogenesis and metabolism.

A key question is whether PLG mediates reverse cholesterol transport from macrophages in vivo. However, reports of its effects on atherosclerosis in animal models are contradictory. In one study, PLG deficiency accelerated atherosclerosis in APOE-deficient mice (52), but another study found reduced atherosclerosis in mice deficient in both APOE and PLG (53). These observations raise the possibility that PLG is involved in multiple pathways implicated in atherogenesis. Future studies must determine whether PLG promotes atherosclerosis and boosts cholesterol efflux from macrophages in animal models.

The Coronary Artery Disease Genome-wide Replication and Meta-analysis Consortium, which analyzed more than 60,000 CVD cases and 130,000 controls, reported that polymorphisms in PLG strongly associated with atherosclerotic vascular disease (49). This observation was confirmed in the latest and largest meta-analysis of genome-wide association for CVD to date (48). Plasma PLG levels were an independent risk factor for coronary artery disease in 2 prospective cohort studies: the FINRISK ' 92 Hemostasis Study (50) and the Atherosclerosis Risk in Communities Study (51). Moreover, PLG remained a risk factor after adjustment for cholesterol and HDL-C levels. However, there was no correlation between plasma and intimal concentrations of PLG in a study of normal and atherosclerotic human intimal tissue harvested at autopsy (54). Those investigators also found that absence of detectable PLG in intimal tissue associated with increased atherosclerosis. In future studies, it will be critical to identify polymorphisms and mutations in PLG that affect the protein's levels in plasma and its ability to promote cholesterol efflux by ABCA1 and, importantly, to determine whether those mutations affect CVD risk in humans. 

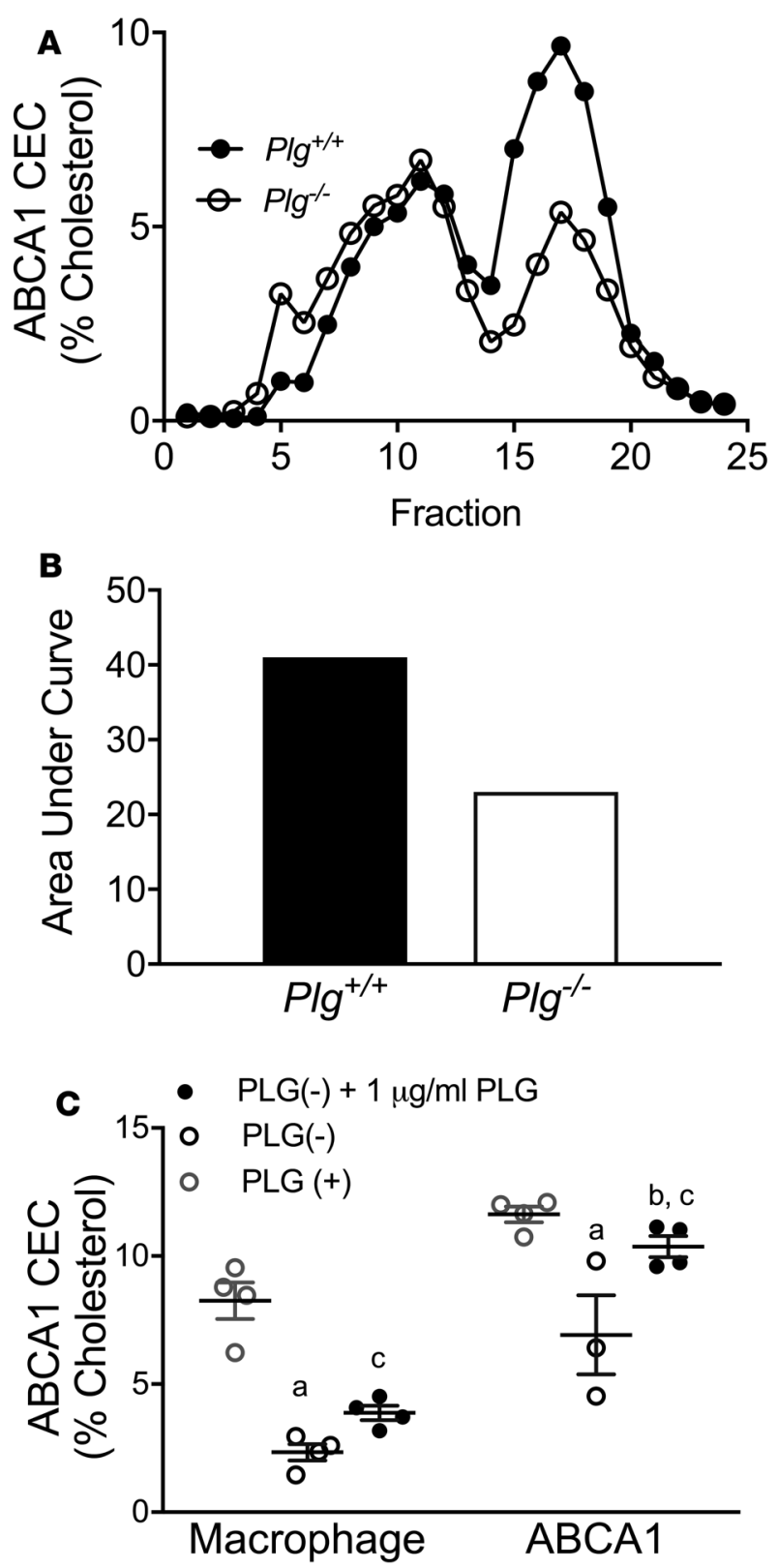

Figure 5. ABCA1 CEC activity of plasma wild-type mice $(\bullet)$ and plasminogen-deficient mice ( $O$ ). ABCA1 cholesterol efflux capacity (CEC) activity was quantified using $\left[{ }^{3} \mathrm{H}\right]$ cholesterol-labeled BHK cells with and without induction of $A B C A 1$ expression.

(A) Plasma fractionated by high-resolution size-exclusion chromatography (SEC). Results are the mean of duplicate determinations of pooled plasma obtained by combining equal volumes of plasma from 5 different mice. (B) Area under the curve for fractions $15-25$ of the data in panel A. HDL eluted in fractions 7-14. (C) Macrophage CEC and ABCA1 CEC of control and plasminogen-depleted (PLG-depleted) human plasma. Four human plasma samples were depleted [PLG(-)], not depleted [PLG(+)], or PLG depleted and supplemented with 1 $\mu \mathrm{g} / \mathrm{ml}$ PLG. PLG was depleted by L-lysine Sepharose affinity chromatography. $P<$ 0.05 for (A) PLG(+) versus PLG(-); (B) PLG(+) versus PLG(-) $+1 \mu \mathrm{g} / \mathrm{ml}$ PLG; (C) PLG(-) versus PLG(-) + $1 \mu \mathrm{g} / \mathrm{ml} \mathrm{PLG}$.

Circulating PLG is a single-chain multidomain glycoprotein of 791 amino acids with 5 kringle domains and a C-terminal trypsin-like serine protease domain (55). We found that treating PLG with PMSF, which inactivates the serine protease, had no effect on the protein's ABCA1 CEC activity. However, 4 of the kringle domains in PLG contain lysine-binding sites (56), and ABCA1 contains 35 lysine residues in extracellular domain 1 and 20 in extracellular domain 2. Chemical modification of these residues abolishes APOA1 binding and sterol efflux that is promoted by $\operatorname{APOA} 1(57,58)$, raising the possibility that lysine-rich regions in extracellular loops of ABCA1 interact with PLG's lysine-binding sites.

PLG has a high degree of sequence homology with Lp(a), an LDL-like lipoprotein whose characteristic protein is LPA, which is strongly linked to cardiovascular disease risk in human clinical and genetic studies (59). In our experiments, however, LPA failed to promote cholesterol efflux from cells by the ABCA1 pathway. Indeed, both LPA and Lp(a) inhibited the ability of PLG — but not APOA1 — to promote cholesterol efflux. Lp(a) upregulates ABCA1 (60), which is consistent with the possibility that the lipoprotein blocks PLG-dependent cholesterol efflux, increasing cellular cholesterol levels and ABCA1 expression. LPA has been proposed to increase CVD risk by 2 mechanisms: altering the prothrombotic/anti-fibrolytic properties of the PLG/plasmin system and promoting intimal deposition of 

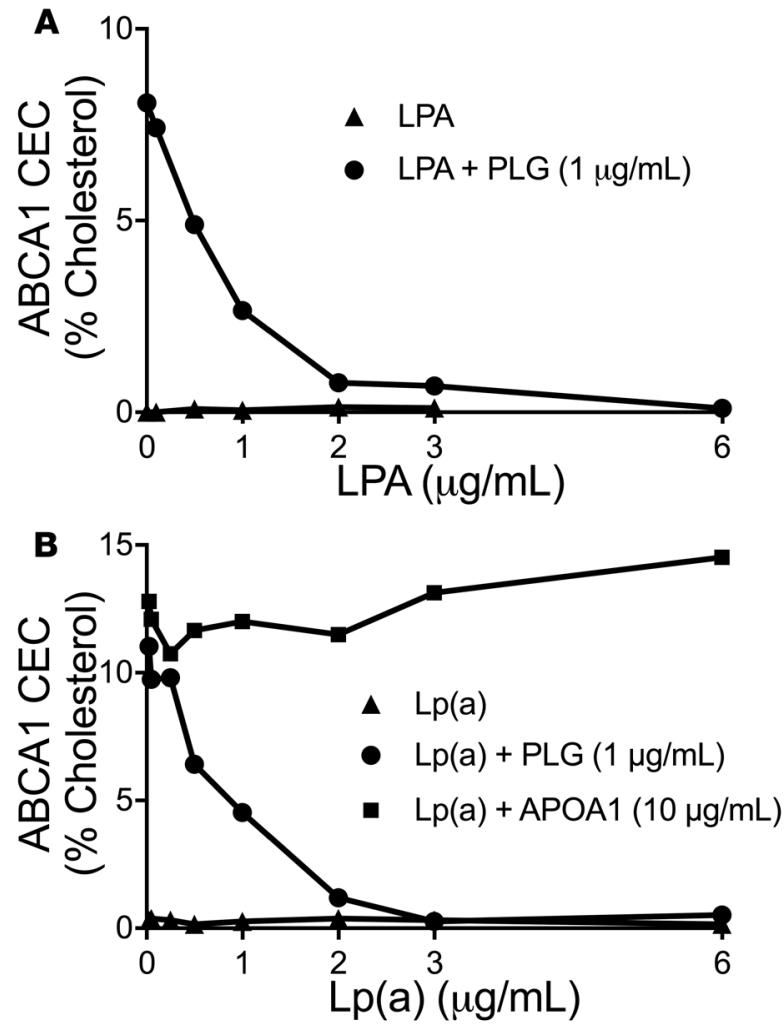

Figure 6. Quantifying the ability of apolipoprotein A (LPA) and lipoprotein(s) to promote cholesterol efflux and to inhibit plasminogen-mediated cholesterol efflux from ABCA1-expressing BHK cells. Cholesterol efflux was quantified using $\left[{ }^{3} \mathrm{H}\right]$ cholesterollabeled BHK cells incubated for 4 hours with the indicated concentrations of lipoprotein(a) [Lp(a)], LPA, or APOA1. Lp(a) was isolated by ultracentrifugation, size-exclusion chromatography, and L-proline affinity chromatography from plasma of a subject with high plasma levels of Lp(a). LPA was isolated from the medium of HEK293 cells expressing human recombinant LPA with 17 kringle domains and purified by L-lysine Sepharose affinity chromatography. CEC, cholesterol efflux capacity; PLG, plasminogen.

Lp(a) cholesterol. Our observations raise the possibility that LPA inhibits PLG-dependent cholesterol efflux, promoting macrophage cholesterol accumulation.

Although APOE-deficient mice have the genetic capacity to synthesize APOA1, the low concentration of HDL particles in these animals strongly suggests that APOE also plays a significant role in HDL assembly or maturation in this animal model. This suggestion is reinforced by the essentially total absence of HDL particles we observed in mice lacking both APOE and APOA1. Despite this anomaly, we still observed measurable levels of cholesterol and phospholipids in the serum HDL of these mice. This likely occurred because these lipids associate with apolipoproteins in serum to form lipoproteins that would not be detected in HDL isolated by ultracentrifugation.

The underlying mechanisms for the marked deficiency of HDL in APOE-deficient mice are unclear, but they may involve altered assembly of HDL particles in the liver or impaired hydrolysis of triglyceriderich lipoproteins that contribute apolipoproteins and phospholipid to HDL formation in plasma. Interestingly, APOA4 deficiency appeared to have little impact on macrophage CEC, ABCA1 CEC, serum HDL cholesterol levels, or HDL particle concentration. The roles of APOE and APOA4 in regulating the distribution of HDL particles merit further study.

In summary, we demonstrated that APOA1 and APOE are major regulators of HDL particle concentration, that $\mathrm{HDL}$ particle concentration is a major determinant of macrophage $\mathrm{CEC}$, but that other proteins are important for promoting ABCA1 CEC. One key factor is PLG, a non-apolipoprotein that stimulates cholesterol efflux by the ABCA1 pathway and is antagonized by LPA and Lp(a). In future studies, it will be important to understand the structural basis and cell biology of PLG-mediated cholesterol efflux and to determine how PLG interacts with ABCA1 to increase cholesterol export. It will also be critical to determine why PLG is a risk factor for CVD in humans.

\section{Methods}

Protein and gene nomenclature. We follow the guidelines of Mouse Genome Informatics and the HUGO Gene Nomenclature Committee. In this system, proteins' names are based on their gene names. Protein names, regardless of species, are in uppercase, not italics. The nomenclature for genes is different: for mouse genes, the first letter is capitalized and all the letters are in italics; for human genes, all the letters 
are capitalized and in italics. For example, the gene name for human apolipoprotein(a) is $L P A$ and the protein name is LPA.

Mice. Studies were approved by the Animal Care and Use committees of the University of Washington, University of Chicago, and Wake Forest University. Transgenic mice were housed (3-5 per cage) in a specific pathogen-free barrier facility $\left(22^{\circ} \mathrm{C}\right)$ with a 12-hour light/dark cycle with free access to food and water. All the strains were fed a low-fat diet (Wayne Rodent BLOX 8604; Harlan Teklad Laboratory) until they were 8-16 weeks old. Mice fasted for 4 hours in the morning were bled from the retro-orbital sinus into tubes containing EDTA (final concentration $1 \mathrm{mM}$ ), and killed by isoflurane inhalation. Plasma samples were collected and stored at $-80^{\circ} \mathrm{C}$ until analysis.

Plasma lipids. Phospholipids (Wako Diagnostics) and cholesterol levels (Invitrogen) were determined biochemically.

$S E C$. Plasma (300 $\mu \mathrm{l})$ was fractionated on a Superdex 200 column ( $24 \mathrm{ml}$ column volume $)$ at a $0.35 \mathrm{ml} /$ min flow rate with PBS, and 0.5 -ml fractions were collected.

Ion-exchange chromatography. The late-eluting peak of material with ABCA1 activity ( $4.5 \mathrm{ml})$ isolated by SEC from 2 pooled plasma samples was concentrated to a final volume of $0.6 \mathrm{ml}$ with 3,000 MW cutoff filters. After passage over a 10DG desalting column (GE Healthcare) equilibrated with buffer A (20 $\mathrm{mM}$ Tris- $\mathrm{HCl}, \mathrm{pH} 8.0$ for MonoQ and $20 \mathrm{mM}$ 2-[N-morpholino] ethanesulfonic acid (MES), $\mathrm{pH} 6.0$ for MonoS) or buffer B (20 mM Tris-HCl, 1.0 M NaCl, pH 8.0 for MonoQ and $20 \mathrm{mM} \mathrm{MES,} 1.0 \mathrm{M} \mathrm{NaCl}$, pH 6.0 for MonoS ), the concentrated material was loaded onto a MonoQ 5/50GL or MonoS 5/50L column (24 $\mathrm{ml}$ volume) at a flow rate of $0.35 \mathrm{ml} / \mathrm{min}$ (61). After the column was washed with $100 \mathrm{ml}$ of buffer A, it was eluted with a linear gradient of $100 \mathrm{ml}$ buffer $\mathrm{B}$, and 0.5 - $\mathrm{ml}$ fractions were collected.

$L p(a)$ isolation. Plasma from 2 subjects with high levels of $\mathrm{Lp}(\mathrm{a})$ was subjected to density gradient ultracentrifugation followed by chromatography on L-proline (62).

Recombinant LPA. Seventeen-kringle LPA expressed by human embryonic kidney cells (63) was isolated from conditioned medium by chromatography on L-lysine Sepharose. Protein purity was assessed by SDSPAGE and silver staining under nonreducing and reducing conditions.

PLG depletion. Human plasma samples were passed through an L-lysine Sepharose column equilibrated with $0.1 \mathrm{M} \mathrm{NaHCO}_{3}$ at $\mathrm{pH} 8.9$ (46). PLG was quantified in samples before and after depletion by immunoblot analysis with a rabbit polyclonal antibody against PLG (Santa Cruz Biotechnology).

Bone marrow-derived macrophages. Bone marrow harvested from femurs and tibias of 16-week-old $\mathrm{Abcal}^{+/+}, \mathrm{Abcal}^{+/-}$, and $\mathrm{Abca1}^{-/-}$mice was gently suspended in RPMI medium (Sigma-Aldrich). Cells were collected by centrifugation ( $300 \mathrm{~g}, 5$ minutes), plated $\left(2 \times 10^{6}\right.$ cells $)$ in 24 -well plates, and cultured for 7 days in RPMI supplemented with $10 \%$ fetal bovine serum, $1 \%$ penicillin, and $30 \mathrm{ng} / \mathrm{ml}$ mouse recombinant M-CSF. Medium was replaced on days 4 and 6. Cells were loaded with cholesterol by incubating them overnight in RPMI medium supplemented with $2 \%$ plasma harvested from LDL receptor-deficient mice fed a high-fat diet for 16 weeks (64-66).

Peritoneal macrophages. Cells were harvested from the peritoneum of C57BL/6J mice 4 days after injection of $1 \mathrm{ml}$ of thioglycolate (Sigma-Aldrich) (67). Cells were washed with PBS, plated, and allowed to adhere for 2 hours at $37^{\circ} \mathrm{C}$ in serum-free high-glucose DMEM (Sigma-Aldrich). Nonadherent cells were removed by 3 washes with PBS. Cells were loaded with cholesterol as described for bone marrow-derived macrophages.

Cholesterol mass. Following the addition of $\left[{ }^{2} \mathrm{H}_{7}\right]$ cholesterol (Sigma-Aldrich) as internal standard and then saponification, lipids were extracted from cell-conditioned medium with hexane and dried under nitrogen gas. Total cholesterol levels were determined after derivatization by LC-MS/MS (68). Free cholesterol was measured with the same procedure without saponification; cholesteryl ester was calculated as total cholesterol minus free cholesterol.

Cholesterol efflux. CEC was assessed with J774 macrophages labeled with $\left[{ }^{3} \mathrm{H}\right]$ cholesterol and stimulated with a cAMP analogue, as described by de la Llera-Moya et al. (24). Efflux by the ABCA1 pathway was measured with BHK cells containing mifepristone-inducible human ABCA1 that were radiolabeled with $\left[{ }^{3} \mathrm{H}\right]$ cholesterol and $1 \mathrm{mg} / \mathrm{ml}$ fatty acid-free albumin (69). Efflux of $\left[{ }^{3} \mathrm{H}\right]$ cholesterol was measured after a 4-hour incubation in medium with or without serum depleted of apoB (2.8\% v/v), human APOA1 (Millipore), human PLG (R\&D Systems), Lp(a), and LPA. Cholesterol efflux was calculated as the percentage of radiolabel in the medium of the cells at the end of the incubation divided by the total radioactivity of the medium and cells. ABCA1-specific cholesterol efflux was monitored as the difference in cholesterol efflux of cells with and without induction of ABCA1. 
Liquid chromatography-electrospray ionization tandem mass spectrometric (LC-ESI-MS/MS) analysis. Proteins $(1 \mu$ g precipitated) were solubilized with $0.1 \%$ RapiGest (Waters) in $200 \mathrm{mM}$ ammonium bicarbonate, reduced with dithiothreitol, alkylated with iodoacetamide, and digested with trypsin (1:20, w/w protein; Promega) for 3 hours at $37^{\circ} \mathrm{C}$. After a second aliquot of trypsin (1:20, w/w protein) was added, samples were incubated overnight at $37^{\circ} \mathrm{C}$. After RapiGest was removed by acid hydrolysis, samples were dried and stored at $-20^{\circ} \mathrm{C}$ until analysis. Prior to analysis, samples were reconstituted in $5 \%$ acetonitrile, $0.1 \%$ formic acid $(42,70)$.

Tryptic digests $(1 \mu \mathrm{g}$ protein) were injected onto a C18 trap column, eluted onto an analytical reverse-phase column $(0.15 \times 150 \mathrm{~mm}$, Magic C18AQ, $5 \mu \mathrm{m}, 200 \mathrm{~A}$; Michrom Bioresources Inc.), and separated on a Paradigm MS4B HPLC (Michrom Bioresources Inc.) (42, 70). Peptides were introduced into the gas phase by electrospray ionization, and positive-ion mass spectra were acquired with an Orbitrap Mass Spectrometer (Thermo Fisher Scientific), using data-dependent acquisition (1 MS survey scan followed by MS/MS scans of the 8 most abundant ions in the survey scan). An exclusion window of 45 seconds was used after 2 acquisitions of the same precursor ion with a mass window $400-2,000 \mathrm{~m} / \mathrm{z}$.

Protein identification. MS/MS spectra were matched against the mouse International Protein Index (IPI) database (mouse v.3.54, Jan 2009), using the SEQUEST (v 2.7) search engine with fixed Cys carbamidomethylation and variable Met oxidation modifications. The mass tolerance for precursor ions was $2.5 \mathrm{Da}$; SEQUEST default tolerance was accepted for product ions. SEQUEST results were further validated with PeptideProphet and ProteinProphet $(71,72)$, using an adjusted probability of 0.90 or higher for peptides and 0.95 or higher for proteins, giving an estimated false discovery rate of $5 \%$. Each charge state of a peptide was considered a unique identification.

We used the gene names for the proteins identified in HDL to eliminate the redundant identifications of isoforms and protein fragments frequently found in protein databases used in proteomic analysis (73). This approach also permits cross-referencing of proteins from different species.

Protein quantification. Proteins were quantified using spectral counts - the total number of MS/ MS spectra detected for a protein. Spectral counting of HDL proteins strongly agrees with the targeted proteomic method, selected reaction monitoring (43, 74-77). Proteins considered for analysis had to be detected in 3 or more analyses with 2 or more unique peptides. When MS/MS spectra could not differentiate between protein isoforms, the isoform with the most unique peptides was used for further analysis. Spectral counts for each protein normalized to total spectral counts for peptides from each sample were used to calculate a spectral index. We used the index to compare relative protein composition (43).

$H D L-P_{I M A}$. HDL-P ${ }_{\text {IMA }}$ was quantified by calibrated ion mobility analysis (38). HDL peak areas were converted into aqueous particle concentrations, using glucose oxidase calibration curves. The method yields a stoichiometry of APOA1 and sizes and relative abundances of HDL subspecies that are in excellent agreement with those determined by nondenaturing gradient gel electrophoresis and analytical ultracentrifugation $(38,78)$.

Statistics. Data are the mean \pm SD. Differences among groups were assessed with ANOVA with Tukey's post-hoc analysis to correct for multiple comparisons. Linear correlations were assessed with Pearson's product-moment coefficient. All reported $P$ values are 2-tailed, with a $P$ value less than 0.05 indicating statistical significance. Analyses were performed with Prism (GraphPad) and R GNU software.

\section{Author contributions}

NP designed studies, conducted experiments, analyzed data, and helped prepare the manuscript. PMH conducted experiments. GER acquired data. HW conducted experiments and acquired data. CT provided reagents. RD, EP, and RW provided mice. TV designed studies and acquired data. VS provided human samples. MLK provided 17 kringle recombinant Apo(a). CAR and GSG provided mice and helped prepare the manuscript. DAD designed studies and helped prepare the manuscript. SM provided reagents, designed studies, and helped prepare the manuscript. JWH designed and analyzed studies and helped prepare the manuscript.

\section{Acknowledgments}

This work was supported by awards from the National Institutes of Health (HL112625, HL108897, P30 DK17047, P01 HL092969, T32HL007828, HL076491). We thank Marlys Koschinsky for providing recombinant 17-kringle LPA. 
Address correspondence to: Nathalie Pamir, Knight Cardiovascular Institute, Oregon Health and Science University, 3181 S.W. Sam Jackson Park Road, Portland, Oregon 97239, USA. Phone: 503.346.1568; Email: pamir@ohsu.edu.

1. Gordon DJ, Rifkind BM. High-density lipoprotein--the clinical implications of recent studies. N Engl J Med. 1989;321(19):1311-1316.

2. Wilson PW, Abbott RD, Castelli WP. High density lipoprotein cholesterol and mortality. The Framingham Heart Study. Arteriosclerosis. 1988;8(6):737-741.

3. Tall AR, Costet P, Wang N. Regulation and mechanisms of macrophage cholesterol efflux. J Clin Invest. 2002;110(7):899-904.

4. Getz GS, Reardon CA. Animal models of atherosclerosis. Arterioscler Thromb Vasc Biol. 2012;32(5):1104-1115

5. Hutchins PM, Heinecke JW. Cholesterol efflux capacity, macrophage reverse cholesterol transport and cardioprotective HDL. Curr Opin Lipidol. 2015;26(5):388-393.

6. AIM-HIGH Investigators, et al. Niacin in patients with low HDL cholesterol levels receiving intensive statin therapy. $N$ Engl $J$ Med. 2011;365(24):2255-2267.

7. HPS2-THRIVE Collaborative Group, et al. Effects of extended-release niacin with laropiprant in high-risk patients. $N$ Engl $J$ Med. 2014;371(3):203-212.

8. Schwartz GG, et al. Effects of dalcetrapib in patients with a recent acute coronary syndrome. N Engl J Med. 2012;367(22):2089-2099.

9. Rader DJ, Hovingh GK. HDL and cardiovascular disease. Lancet. 2014;384(9943):618-625.

10. Kingwell BA, Chapman MJ, Kontush A, Miller NE. HDL-targeted therapies: progress, failures and future. Nat Rev Drug Discov. 2014;13(6):445-464.

11. van Capelleveen JC, Brewer HB, Kastelein JJ, Hovingh GK. Novel therapies focused on the high-density lipoprotein particle. Circ Res. 2014;114(1):193-204.

12. Vickers KC, Remaley AT. HDL and cholesterol: life after the divorce? J Lipid Res. 2014;55(1):4-12.

13. Zanoni P, et al. Rare variant in scavenger receptor BI raises HDL cholesterol and increases risk of coronary heart disease. Science. 2016;351(6278):1166-1171.

14. Zhang S, et al. Diet-induced occlusive coronary atherosclerosis, myocardial infarction, cardiac dysfunction, and premature death in scavenger receptor class B type I-deficient, hypomorphic apolipoprotein ER61 mice. Circulation. 2005;111(25):3457-3464.

15. Rader DJ, Alexander ET, Weibel GL, Billheimer J, Rothblat GH. The role of reverse cholesterol transport in animals and humans and relationship to atherosclerosis. J Lipid Res. 2009;50 Suppl:S189-S194.

16. Tall AR, Yvan-Charvet L, Terasaka N, Pagler T, Wang N. HDL, ABC transporters, and cholesterol efflux: implications for the treatment of atherosclerosis. Cell Metab. 2008;7(5):365-375.

17. Oram JF, Heinecke JW. ATP-binding cassette transporter A1: a cell cholesterol exporter that protects against cardiovascular disease. Physiol Rev. 2005;85(4):1343-1372.

18. Rye KA, Barter PJ. Regulation of high-density lipoprotein metabolism. Circ Res. 2014;114(1):143-156.

19. Rothblat GH, Phillips MC. High-density lipoprotein heterogeneity and function in reverse cholesterol transport. Curr Opin Lipidol. 2010;21(3):229-238.

20. Bashtovyy D, Jones MK, Anantharamaiah GM, Segrest JP. Sequence conservation of apolipoprotein A-I affords novel insights into HDL structure-function. J Lipid Res. 2011;52(3):435-450.

21. van Eck M, et al. Leukocyte ABCA1 controls susceptibility to atherosclerosis and macrophage recruitment into tissues. Proc Natl Acad Sci USA. 2002;99(9):6298-6303.

22. Van Eck M, et al. Macrophage ATP-binding cassette transporter A1 overexpression inhibits atherosclerotic lesion progression in low-density lipoprotein receptor knockout mice. Arterioscler Thromb Vasc Biol. 2006;26(4):929-934.

23. Ferrans VJ, Fredrickson DS. The pathology of Tangier disease. A light and electron microscopic study. Am J Pathol. 1975;78(1):101-158.

24. de la Llera-Moya M, Drazul-Schrader D, Asztalos BF, Cuchel M, Rader DJ, Rothblat GH. The ability to promote efflux via $\mathrm{ABCA} 1$ determines the capacity of serum specimens with similar high-density lipoprotein cholesterol to remove cholesterol from macrophages. Arterioscler Thromb Vasc Biol. 2010;30(4):796-801.

25. Khera AV, et al. Cholesterol efflux capacity, high-density lipoprotein function, and atherosclerosis. $N$ Engl J Med. 2011;364(2):127-135.

26. Rohatgi A, et al. HDL cholesterol efflux capacity and incident cardiovascular events. N Engl J Med. 2014;371(25):2383-2393

27. Saleheen D, et al. Association of HDL cholesterol efflux capacity with incident coronary heart disease events: a prospective case-control study. Lancet Diabetes Endocrinol. 2015;3(7):507-513.

28. Sankaranarayanan S, et al. A sensitive assay for ABCA1-mediated cholesterol efflux using BODIPY-cholesterol. J Lipid Res. 2011;52(12):2332-2340.

29. Remaley AT, Schumacher UK, Stonik JA, Farsi BD, Nazih H, Brewer HB. Decreased reverse cholesterol transport from Tangier disease fibroblasts. Acceptor specificity and effect of brefeldin on lipid efflux. Arterioscler Thromb Vasc Biol. 1997;17(9):1813-1821.

30. Wang X, et al. Macrophage ABCA1 and ABCG1, but not SR-BI, promote macrophage reverse cholesterol transport in vivo. J Clin Invest. 2007;117(8):2216-2224.

31. Yvan-Charvet L, et al. Combined deficiency of ABCA1 and ABCG1 promotes foam cell accumulation and accelerates atherosclerosis in mice. J Clin Invest. 2007;117(12):3900-3908.

32. Shao B, et al. Myeloperoxidase impairs ABCA1-dependent cholesterol efflux through methionine oxidation and site-specific tyrosine chlorination of apolipoprotein A-I. J Biol Chem. 2006;281(14):9001-9004.

33. Fournier N, et al. Human ApoA-IV overexpression in transgenic mice induces cAMP-stimulated cholesterol efflux from J774 macrophages to whole serum. Arterioscler Thromb Vasc Biol. 2000;20(5):1283-1292.

34. von Eckardstein A, et al. Lipoproteins containing apolipoprotein A-IV but not apolipoprotein A-I take up and esterify cellderived cholesterol in plasma. Arterioscler Thromb Vasc Biol. 1995;15(10):1755-1763. 
35. Plump AS, Breslow JL. Apolipoprotein E and the apolipoprotein E-deficient mouse. Annu Rev Nutr. 1995;15:495-518.

36. Voyiaziakis E, Goldberg IJ, Plump AS, Rubin EM, Breslow JL, Huang LS. ApoA-I deficiency causes both hypertriglyceridemia and increased atherosclerosis in human apoB transgenic mice. J Lipid Res. 1998;39(2):313-321.

37. Linton MF, Atkinson JB, Fazio S. Prevention of atherosclerosis in apolipoprotein E-deficient mice by bone marrow transplantation. Science. 1995;267(5200):1034-1037.

38. Hutchins PM, et al. Quantification of HDL particle concentration by calibrated ion mobility analysis. Clin Chem. 2014;60(11):1393-1401.

39. Pamir N, et al. Proteomic analysis of HDL from inbred mouse strains implicates APOE associated with HDL in reduced cholesterol efflux capacity via the ABCA1 pathway. J Lipid Res. 2016;57(2):246-257.

40. Sankaranarayanan S, de la Llera-Moya M, Drazul-Schrader D, Phillips MC, Kellner-Weibel G, Rothblat GH. Serum albumin acts as a shuttle to enhance cholesterol efflux from cells. J Lipid Res. 2013;54(3):671-676.

41. Gundry RL, Fu Q, Jelinek CA, Van Eyk JE, Cotter RJ. Investigation of an albumin-enriched fraction of human serum and its albuminome. Proteomics Clin Appl. 2007;1(1):73-88.

42. Vaisar T, et al. Shotgun proteomics implicates protease inhibition and complement activation in the antiinflammatory properties of HDL. J Clin Invest. 2007;117(3):746-756.

43. Ronsein GE, et al. Parallel reaction monitoring (PRM) and selected reaction monitoring (SRM) exhibit comparable linearity, dynamic range and precision for targeted quantitative HDL proteomics. J Proteomics. 2015;113:388-399.

44. Vaughan AM, Oram JF. ABCG1 redistributes cell cholesterol to domains removable by high density lipoprotein but not by lipiddepleted apolipoproteins. J Biol Chem. 2005;280(34):30150-30157.

45. Sekar V, Hageman JH. Specificity of the serine protease inhibitor, phenylmethylsulfonyl fluoride. Biochem Biophys Res Commun 1979;89(2):474-478.

46. Deutsch DG, Mertz ET. Plasminogen: purification from human plasma by affinity chromatography. Science. 1970;170(3962):1095-1096.

47. Marcovina SM, Koschinsky ML. Lipoprotein(a) as a risk factor for coronary artery disease. Am J Cardiol. 1998;82(12A):57U-66U.

48. Nikpay M, et al. A comprehensive 1,000 Genomes-based genome-wide association meta-analysis of coronary artery disease. Nat Genet. 2015;47(10):1121-1130.

49. CARDIoGRAMplusC4D Consortium, et al. Large-scale association analysis identifies new risk loci for coronary artery disease Nat Genet. 2013;45(1):25-33.

50. Rajecki M, Pajunen P, Jousilahti P, Rasi V, Vahtera E, Salomaa V. Hemostatic factors as predictors of stroke and cardiovascular diseases: the FINRISK '92 Hemostasis Study. Blood Coagul Fibrinolysis. 2005;16(2):119-124.

51. Folsom AR, Aleksic N, Park E, Salomaa V, Juneja H, Wu KK. Prospective study of fibrinolytic factors and incident coronary heart disease: the Atherosclerosis Risk in Communities (ARIC) Study. Arterioscler Thromb Vasc Biol. 2001;21(4):611-617.

52. Xiao Q, et al. Plasminogen deficiency accelerates vessel wall disease in mice predisposed to atherosclerosis. Proc Natl Acad Sci USA. 1997;94(19):10335-10340.

53. Kremen M, et al. Plasminogen mediates the atherogenic effects of macrophage-expressed urokinase and accelerates atherosclerosis in apoE-knockout mice. Proc Natl Acad Sci USA. 2008;105(44):17109-17114.

54. Smith EB, Ashall C. Fibrinolysis and plasminogen concentration in aortic intima in relation to death following myocardial infarction. Atherosclerosis. 1985;55(2):171-186.

55. Petersen TE, Martzen MR, Ichinose A, Davie EW. Characterization of the gene for human plasminogen, a key proenzyme in the fibrinolytic system. J Biol Chem. 1990;265(11):6104-6111.

56. Xue Y, Bodin C, Olsson K. Crystal structure of the native plasminogen reveals an activation-resistant compact conformation. J Thromb Haemost. 2012;10(7):1385-1396.

57. Nagao K, Kimura Y, Ueda K. Lysine residues of ABCA1 are required for the interaction with apoA-I. Biochim Biophys Acta. 2012;1821(3):530-535.

58. Sorci-Thomas MG, Thomas MJ. Microdomains, inflammation, and atherosclerosis. Circ Res. 2016;118(4):679-691.

59. Emerging Risk Factors Collaboration, et al. Lipoprotein(a) concentration and the risk of coronary heart disease, stroke, and nonvascular mortality. JAMA. 2009;302(4):412-423.

60. Sharma M, Von Zychlinski-Kleffmann A, Porteous CM, Jones GT, Williams MJ, McCormick SP. Lipoprotein (a) upregulates ABCA1 in liver cells via scavenger receptor-B1 through its oxidized phospholipids. J Lipid Res. 2015;56(7):1318-1328.

61. Gherardi E, Gray J, Stoker M, Perryman M, Furlong R. Purification of scatter factor, a fibroblast-derived basic protein that modulates epithelial interactions and movement. Proc Natl Acad Sci USA. 1989;86(15):5844-5848.

62. Marcovina SM, et al. Use of a reference material proposed by the International Federation of Clinical Chemistry and Laboratory Medicine to evaluate analytical methods for the determination of plasma lipoprotein(a). Clin Chem. 2000;46(12):1956-1967.

63. Romagnuolo R, Marcovina SM, Boffa MB, Koschinsky ML. Inhibition of plasminogen activation by apo(a): role of carboxylterminal lysines and identification of inhibitory domains in apo(a). J Lipid Res. 2014;55(4):625-634.

64. Lutz MB, et al. An advanced culture method for generating large quantities of highly pure dendritic cells from mouse bone marrow. J Immunol Methods. 1999;223(1):77-92.

65. Inaba K, et al. Generation of large numbers of dendritic cells from mouse bone marrow cultures supplemented with granulocyte/macrophage colony-stimulating factor. J Exp Med. 1992;176(6):1693-1702.

66. Pamir N, et al. Granulocyte/macrophage colony-stimulating factor-dependent dendritic cells restrain lean adipose tissue expansion. J Biol Chem. 2015;290(23):14656-14667.

67. Becker L, et al. Unique proteomic signatures distinguish macrophages and dendritic cells. PLoS One. 2012;7(3):e33297.

68. Honda A, et al. Highly sensitive quantification of key regulatory oxysterols in biological samples by LC-ESI-MS/MS. $J$ Lipid Res. 2009;50(2):350-357.

69. Shao B, et al. Acrolein impairs ATP binding cassette transporter A1-dependent cholesterol export from cells through site-specific modification of apolipoprotein A-I. J Biol Chem. 2005;280(43):36386-36396

70. Cheung MC, Vaisar T, Han X, Heinecke JW, Albers JJ. Phospholipid transfer protein in human plasma associates with proteins linked to immunity and inflammation. Biochemistry. 2010;49(34):7314-7322. 
71. Keller A, Nesvizhskii AI, Kolker E, Aebersold R. Empirical statistical model to estimate the accuracy of peptide identifications made by MS/MS and database search. Anal Chem. 2002;74(20):5383-5392.

72. Nesvizhskii AI, Keller A, Kolker E, Aebersold R. A statistical model for identifying proteins by tandem mass spectrometry. Anal Chem. 2003;75(17):4646-4658.

73. Rappsilber J, Mann M. What does it mean to identify a protein in proteomics? Trends Biochem Sci. 2002;27(2):74-78.

74. Liu H, Sadygov RG, Yates JR. A model for random sampling and estimation of relative protein abundance in shotgun proteomics. Anal Chem. 2004;76(14):4193-4201.

75. Old WM, et al. Comparison of label-free methods for quantifying human proteins by shotgun proteomics. Mol Cell Proteomics. 2005;4(10):1487-1502.

76. Fu X, et al. Spectral index for assessment of differential protein expression in shotgun proteomics. J Proteome Res. 2008;7(3):845-854.

77. Hoofnagle AN, Becker JO, Oda MN, Cavigiolio G, Mayer P, Vaisar T. Multiple-reaction monitoring-mass spectrometric assays can accurately measure the relative protein abundance in complex mixtures. Clin Chem. 2012;58(4):777-781.

78. Rosenson RS, et al. HDL measures, particle heterogeneity, proposed nomenclature, and relation to atherosclerotic cardiovascular events. Clin Chem. 2011;57(3):392-410. 